\title{
Disseminated alveolar echinococcosis in a 74-year-old woman presenting with focal seizure
}

\author{
Christopher J. Olivier MD, Hao Li MD, Roland N. Auer MD PhD, Devika Dixit MSc MD
}

Cite as: CMAJ 2019 August 26;191:E940-3. doi: 10.1503/cmaj.181258

A 74-year-old woman with type 2 diabetes mellitus presented to hospital following new-onset focal seizure characterized by right-sided facial droop and numbness, with spread to the right eye and slurred speech. These symptoms resolved before she presented to hospital. Neuroimaging with computed tomography (CT), followed by magnetic resonance imaging (MRI), showed a dural-based, gadolinium-enhancing lesion in her left frontal lobe, measuring $28 \times 12 \times 28 \mathrm{~mm}^{3}$ with a multicystic appearance and substantial surrounding edema (Figure 1A). As part of a malignancy workup, ultrasonography, CT and MRI of the abdomen and pelvis showed multiple small cystic lesions within the liver, spleen, pancreas and kidneys. On ultrasonography of the abdomen, the splenic cysts showed internal hyperechoic components; hepatic cysts were benign in appearance (Figure 2). Blood work included a normal complete blood count with a white blood cell count of $3.81 \times 10^{9} / \mathrm{L}$ and an absolute eosinophil count of 0.12 (normal range $0.00-0.60$ ). Her liver enzymes were elevated, with aspartate aminotransferase 62 (normal range 14-36) $\mathrm{U} / \mathrm{L}$, alanine aminotransferase 52 (normal range 9-52) U/L, alkaline phosphatase 173 (normal range 38-126) U/L, and $\gamma$-glutamyl transferase 301 (normal range 12-43) U/L.

Dexamethasone and phenytoin were prescribed. An electroencephalogram performed while the patient was taking phenytoin showed focal left temporal slowing in keeping with the underlying structural abnormality. The working diagnosis at the time was neoplasm (primary versus metastatic); an infectious etiology was considered to be less likely.

An open diagnostic biopsy of the cranial lesion was performed, although a minimal amount of tissue was sampled owing to proximity to the primary motor cortex. Pathology showed hypertrophic meninges, suggesting a meningioma. The biopsy was complicated by the development of mild, nonresolving, right-sided weakness in the patient. The patient was discharged 2 days after the open biopsy.

Two weeks postdischarge, the patient was readmitted to hospital because of worsening right hemiplegia. Magnetic resonance imaging of the brain showed about threefold increase in the size of the brain lesion, now measuring $33 \times 28 \times 33 \mathrm{~mm}^{3}$ (previously $28 \times 12 \times 28 \mathrm{~mm}^{3}$ ) with increased edema (Figure 1B).

\section{KEY POINTS}

- Alveolar echinococcosis is an aggressive infection caused by the cestode Echinococcus multilocularis and most commonly affects the liver; metastatic or disseminated disease can occur but are uncommon.

- Although alveolar echinococcosis is endemic in Canada, the epidemiology is not fully characterized; E. multilocularis is also found worldwide, in particular, Europe and Asia.

- New evidence suggests an expanding geographic distribution of the parasite in animal populations within Canada.

- Human alveolar echinococcosis requires prolonged or repeated exposure to infected animals such as foxes, coyotes, wolves, dogs or cats.

- Diagnosis of alveolar echinococcosis requires a history of compatible exposure, congruent imaging and histopathologic or molecular confirmation.

- Treatment of $E$. multilocularis infection involves radical surgical resection when possible and prolonged antiparasitic pharmacotherapy with albendazole (benzimidazoles); untreated infection has very high mortality.

A decision to operate emergently was made given the patient's decline, rapid increase in size of the lesion and an unclear etiology not in keeping with a meningioma; a near-complete surgical excision was performed.

Intraoperatively, the mass was found to be infiltrating the brain. On gross inspection, the specimen consisted of multilocular cystic lesions, each 1-3 mm in diameter, containing a granular cottage cheese-like material (Figure 3). Microscopically, embedded within the brain tissue were numerous cysts containing larval parts that were mostly necrotic. Staining with Ziehl-Neelsen showed uncommon degenerate hooklets of protoscolices. The brain tissue surrounding the larval cysts was largely necrotic, with a massive amount of inflammation consisting of eosinophils, neutrophils, lymphocytes, plasma cells and histiocytes (Appendix 1, available at www.cmaj.ca/lookup/suppl/doi:10.1503/ cmaj.181258/-/DC1). Initial pathology was reported as neurocysticercosis, but this observation did not align clearly with neuroimaging, which did not show the scolices typical of the disease. 

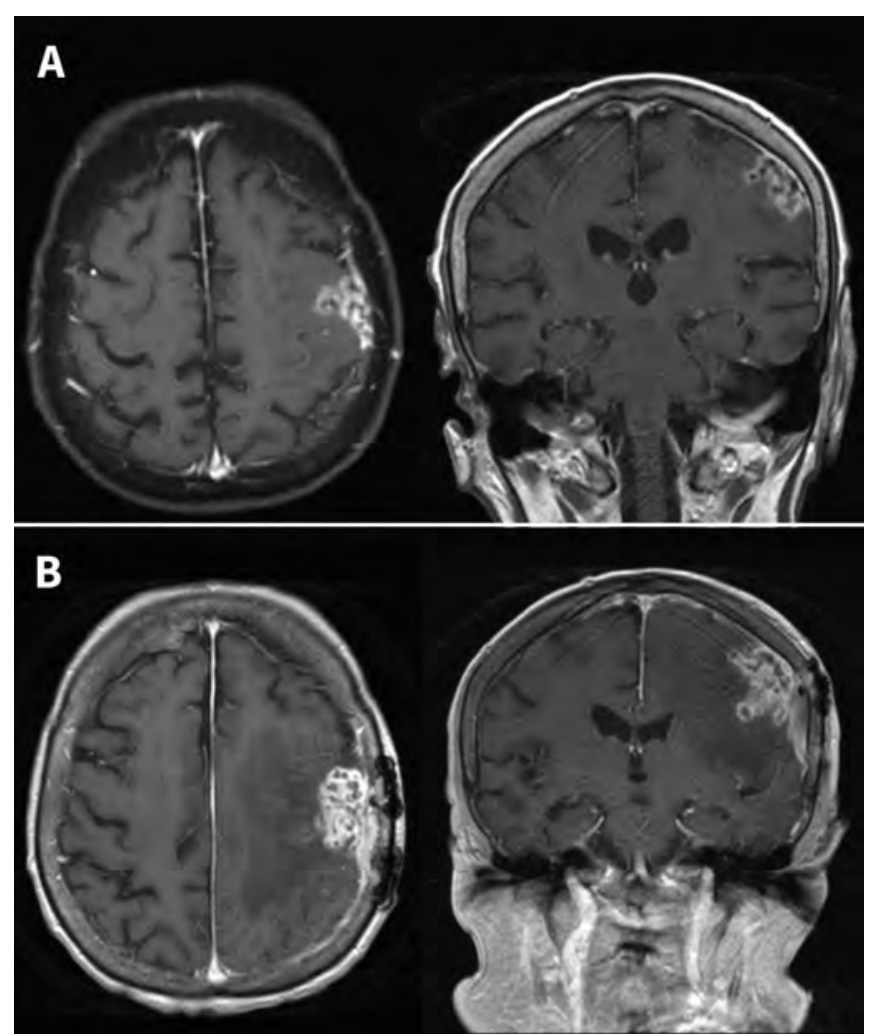

Figure 1: Magnetic resonance imaging of the brain of a 74-year-old woman with new onset of neurologic deficits, $T_{1}$-weighted with gadolinium contrast, showing a dural-based multicystic lesion in the left frontal lobe (A) on initial presentation and (B) after 1 month, with increased size and edema postbiopsy.
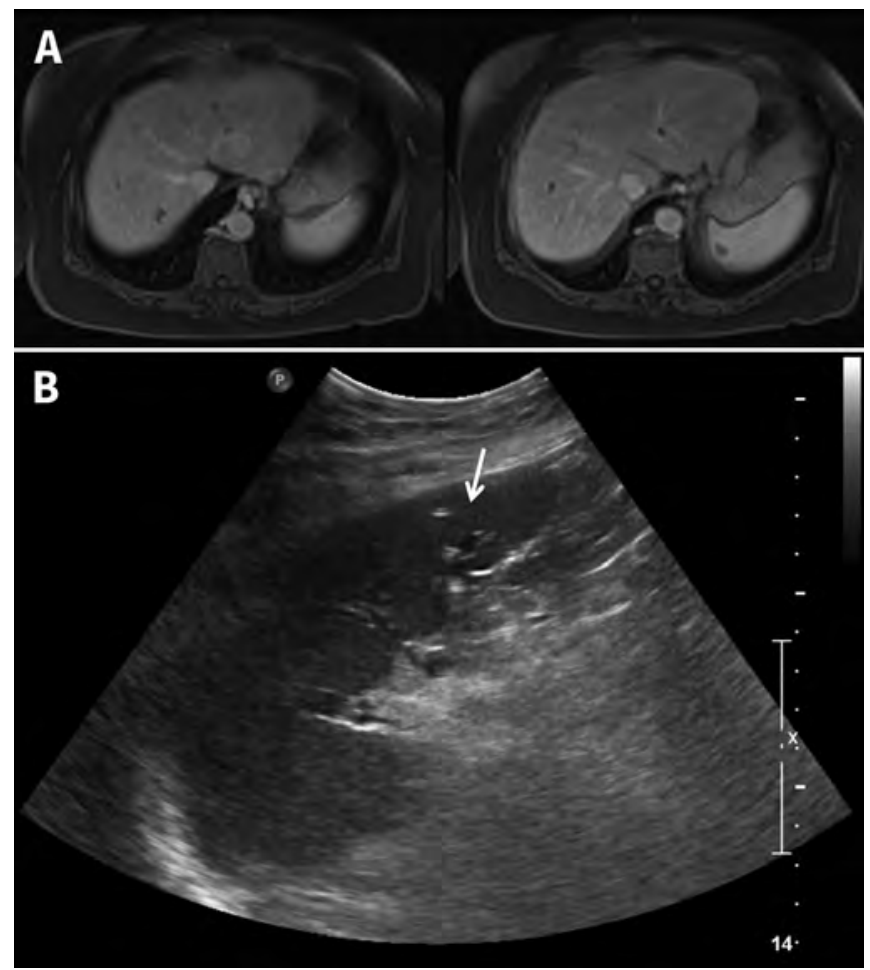

Figure 2: (A) Magnetic resonance imaging of the abdomen showing multiple cysts in the abdominal organs. (B) Ultrasound of the spleen showing a cyst with multiple hyperechoic components (arrow).
On review of relevant risk factors, our patient did not have any history of travel outside her home province of Saskatchewan, where she and her family had resided on a mixed farm containing cows and pigs. Her family members hunted wild game locally, accompanied by several hunting dogs. She had had regular contact with the dogs over the preceding decade.

We requested additional studies from the National Reference Centre for Parasitology at the J.D. MacLean Centre for Tropical Diseases at McGill University. Serum enzyme-linked immunosorbent assay was weakly positive for Echinococcus antigens at an optical density of 0.80 . Tissue polymerase chain reaction of the brain lesion was positive for Echinococcus multilocularis.

An MRI of our patient's abdomen, repeated at the start of therapy, showed no change in the size of the previously described intra-abdominal cysts. A transthoracic echocardiogram to rule out cardiac echinococcosis and a cardiac shunt in the setting of central nervous system alveolar echinococcosis was negative.

We prescribed albendazole $400 \mathrm{mg}$ orally twice daily indefinitely, with serial monitoring of complete blood cell count, liver enzymes and abdominal imaging. After the patient began medical therapy, we discharged her to inpatient rehabilitation for the neurologic deficits. An MRI of her brain 2 months after resection showed resolution of mass effect with a small amount of residual disease (Appendix 2, available at www.cmaj.ca/lookup/suppl/ doi:10.1503/cmaj.181258/-/DC1). Unfortunately, our patient died 8 months after her initial presentation, of an unknown cause while in hospital; an autopsy was not performed.

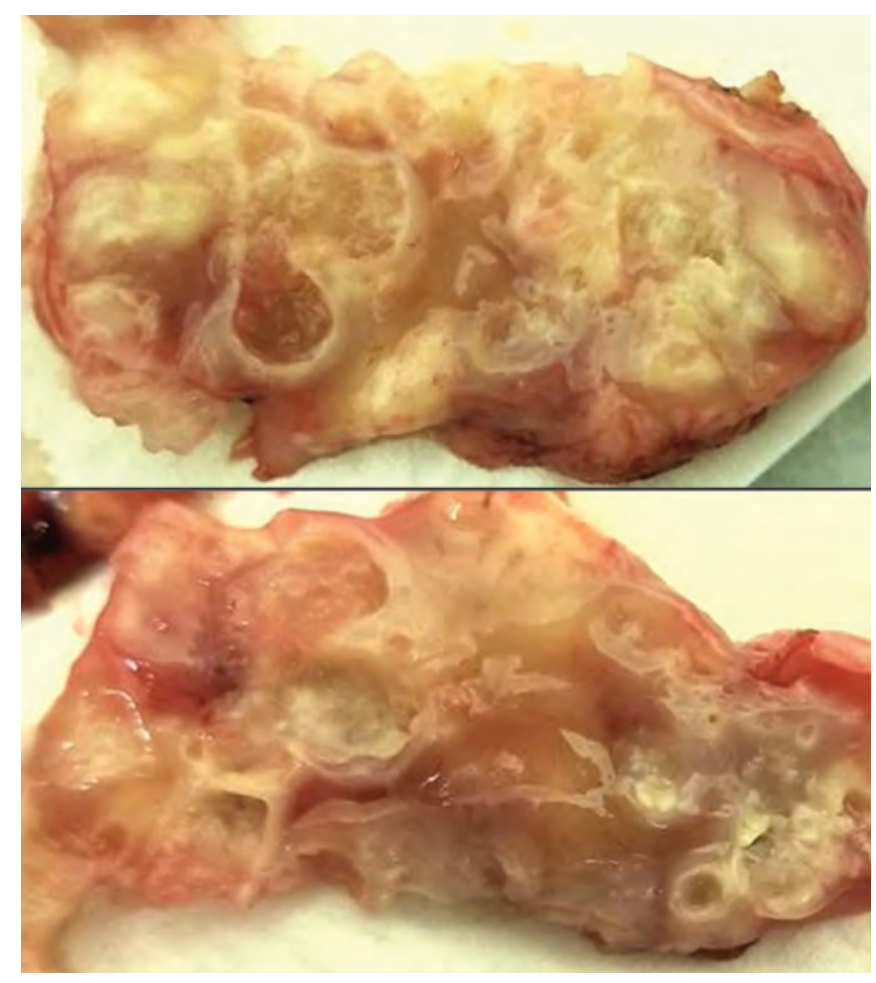

Figure 3: Fragments of the gross surgical specimen of the brain, composed of multiple cysts with a caseous appearance, consistent with Echinococcus multilocularis infection. 


\section{Discussion}

We present a case of alveolar echinococcosis with histopathologic and tissue nucleic acid confirmation of brain involvement from disseminated and metastatic spread. In our patient, exposure to E. multilocularis is thought to have occurred after prolonged contact to family hunting dogs, which were presumably infected by ingesting an intermediate host of the organism. It is unclear why this patient developed disseminated disease, as no cardiac disease or shunt was discovered. Despite elevated liver enzymes, the hepatic lesions did not have characteristic features of alveolar echinococcosis on imaging. The splenic lesions were the only abdominal cystic structures with imaging features typical for alveolar echinococcosis.

The patient experienced worsening of central nervous system disease from protoscolex spillage from initial sampling of the brain lesion without concurrent or postinterventional anthelmintic pharmacotherapy.

\section{Pathobiology}

Adult cestodes of E. multilocularis residing in the intestines of the definitive host (foxes, coyotes, wolves and domesticated dogs and cats) produce eggs that are excreted in feces (Figure 4). Eggs are ingested by the intermediate host (small rodents) and then hatch in their small intestines and release an oncosphere. The oncosphere penetrates the intestinal wall and disseminates into tissues (most often the liver), forming cysts containing protoscolices and daughter cysts. The definitive host becomes infected after ingesting cysts contained in the organs of the intermediate host. Protoscolices evaginate within the intestines of the definitive host and produce eggs after maturing into their adult form. Humans are considered aberrant intermediate hosts who can develop infection with the larval form of E. multilocularis after accidental ingestion of eggs $^{1}$ (the presumed source of infection in our patient).

Prevention of alveolar echinococcosis in human populations involves avoidance of typical definitive hosts in endemic areas of $E$. multilocularis, eradication of disease in infected domesticated

\section{(3DPDx

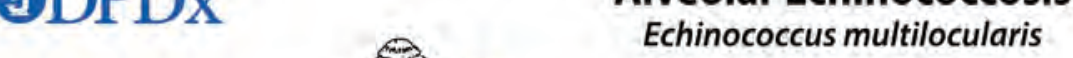 \\ Alveolar Echinococcosis Echinococcus multilocularis}

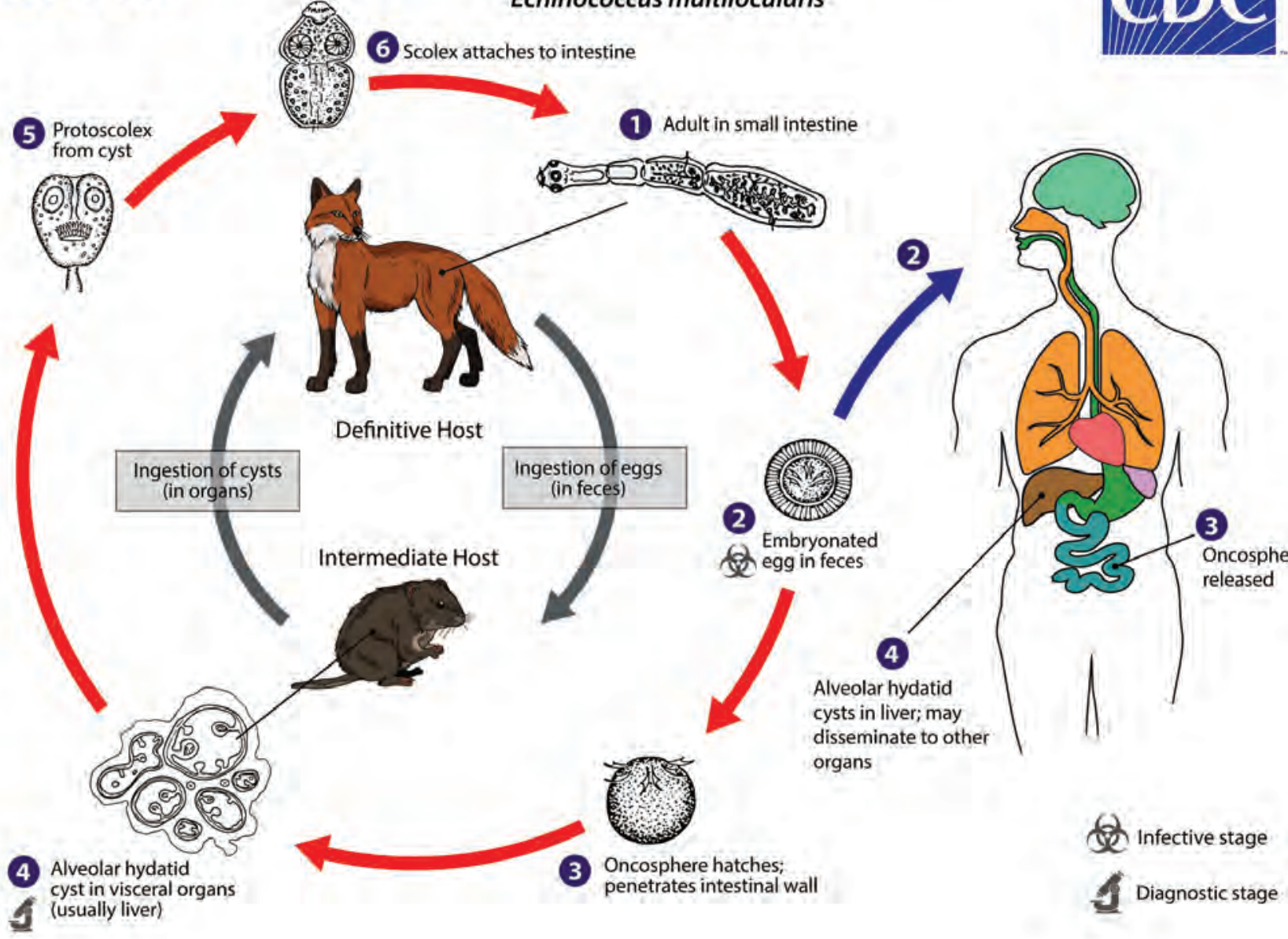

Figure 4: Life cycle of Echinococcus multilocularis. Image courtesy of DPDx, Centers for Disease Control and Prevention (www.cdc.gov/dpdx/echinococcosis/ index.html). 
hosts (e.g., dogs) and proper hand hygiene (www.cdc. gov/parasites/echinococcosis/gen_info/ae-faqs.html and www. cdc.gov/parasites/echinococcosis/prevent.html).

\section{Epidemiology}

Echinococcus multilocularis is known to be endemic to the Canadian Arctic and the southern areas of Manitoba, Saskatchewan and Alberta, as well as in Europe and Asia. Recent evidence also shows high prevalence in wild canids in southern Ontario. This area was not previously shown to be endemic for the pathogen, and suggests a possible change in geographic distribution. ${ }^{2}$ Despite this, the annual incidence of human alveolar echinococcosis in endemic regions is believed to be low (0.03-1.2 cases per 100000 inhabitants). ${ }^{3}$ To our knowledge, 1 published case report of human alveolar echinococcosis acquired in Canada (Winnipeg, Manitoba) exists in the literature, which was published in $1937 .{ }^{4}$ There have been 14 cases (13 confirmed, 1 probable) in Alberta since 2013 (Dr. Stan Houston, Division of Infectious Diseases, Department of Medicine, University of Alberta, Edmonton, Alta.: personal communication, Aug. 9, 2019). 5,6 It may be that the current human prevalence of infection with E. multilocularis is under-recognized in Canada, owing to a lack of mandatory reporting and unfamiliarity of the entity among health care professionals. ${ }^{7}$

\section{Alveolar echinococcosis}

Diagnosis of alveolar echinococcosis is established through the following 3 features: clinical features and exposure history; imaging findings and tissue histopathology; and/or nucleic acid testing and serology. ${ }^{8}$ Signs and symptoms of alveolar echinococcosis most commonly develop after an initial asymptomatic period of 5 to 15 years, and differ depending on site of infection. ${ }^{1}$ The liver is by far the most common primary location of infection (in 99\% of cases), ${ }^{1,9}$ from which infection may extend locally or metastasize via lymphatics or blood vessels to distant sites, such as the spleen and brain. Common imaging features include a partly multivesicular solid mass, occasionally with central necrosis. ${ }^{9}$ Definitive diagnosis can be obtained by sending tissue specimens for histopathology and tissue nucleic acid testing. ${ }^{5,8}$

A classification system for alveolar echinococcosis developed by the World Health Organization Informal Working Group on Echinococcosis categorizes alveolar echinococcosis based on the parasitic mass in the liver $(P)$, involvement of neighbouring organs (N) and metastases (M). ${ }^{3}$ Our patient was classified as P1 (peripheral lesions without proximal vascular or biliary involvement or both), N1 (regional involvement of contiguous organs or tissues) and M1 (metastases). Given her disseminated disease, complete surgical excision was not considered an option and long-term albendazole was necessary.

\section{Treatment}

Treatment of alveolar echinococcosis consists of radical surgical resection whenever possible. Concomitant chemotherapy with albendazole is necessary if radical or nonradical surgery is performed, to prevent protoscolex spillage and dissemination of infection. Patients who receive complete surgical excision should receive a limited course of pharmacotherapy after resection. Incomplete excision necessitates lifelong therapy to limit growth and metastasis of residual lesions. ${ }^{8}$ Prognosis of alveolar echinococcosis has improved considerably over the last 50 years. Historically, 10-year survival rates of alveolar echinococcosis were less than $10 \%$, but have increased to $85 \%-90 \%$ with the use of antihelmintic therapy and improved surgical techniques. ${ }^{3}$

\section{References}

1. Moro P, Schantz PM. Echinococcosis: a review. Int J Infect Dis 2009;13:125-33.

2. Kotwa JD, Isaksson M, Jardine CM, et al. Echinococcus multilocularis infection, Southern Ontario, Canada. Emerg Infect Dis 2019;25:265-72.

3. WHO Informal Working Group on Echinococcosis. Guidelines for treatment of cystic and alveolar echinococcosis in humans. Bull World Health Organ 1996; 74:231-42.

4. James E, Boyd W. Echinococcus alveolaris: (with the report of a case). Can Med Assoc J 1937;36:354-6.

5. Alveolar echinococcosis (E. multilocularis). Edmonton: Alberta Health Services ProvLab; 2018:1-3. Available: www.albertahealthservices.ca/assets/wf/plab/ wf-provlab-alveolar-echinococcosis-e-multilocularis-information.pdf (accessed 2019 Jan. 10).

6. Massolo A, Klein C, Kowalewska-Grochowska K, et al. European Echinococcus multilocularis Identified in patients in Canada. NEJM 2019;381:384-385.

7. Schurer JM, Rafferty E, Farag M, et al. Echinococcosis: an economic evaluation of a veterinary public health intervention in rural Canada. PLoS Negl Trop Dis 2015;9:e0003883.

8. Brunetti E, Kern P, Vuitton DA. Expert consensus for the diagnosis and treatment of cystic and alveolar echinococcosis in humans. Acta Trop 2010;114:1-6.

9. Kern P. Clinical features and treatment of alveolar echinococcosis. Curr Opin Infect Dis 2010;23:505-12.

\section{Competing interests: None declared.}

This article has been peer reviewed.

The authors have obtained patient consent.

Affiliations: Department of Medicine (Olivier, Dixit), and Division of Neurosurgery (Li; during case management), Department of Surgery and Department of Pathology (Auer), College of Medicine; Division of Infectious Diseases (Dixit), Department of Medicine, University of Saskatchewan, Saskatoon, Sask.; Division of Anatomical Pathology (Li), Department of Pathology and Laboratory Medicine, Schulich School of Medicine \& Dentistry, Western University, London, Ont.

Contributors: All of the authors contributed to the conception and design of the work, drafted the manuscript, revised it critically for important intellectual content, gave final approval of the version to be published and agreed to be accountable for all aspects of the work.

Correspondence to: Devika Dixit, ddixit@ualberta.ca

Note [from authors] on figures: Manipulation of the images in this manuscript includes adjustment of brightness and contrast, as well as erasure of irrelevant artifacts (e.g., dust particles).

The section Cases presents brief case reports that convey clear, practical lessons. Preference is given to common presentations of important rare conditions, and important unusual presentations of common problems. Articles start with a case presentation (500 words maximum), and a discussion of the underlying condition follows (1000 words maximum). Visual elements (e.g., tables of the differential diagnosis, clinical features or diagnostic approach) are encouraged. Consent from patients for publication of their story is a necessity. See information for authors at www.cmaj.ca. 